\title{
Current and Future Trends in Game-Based Learning
}

\author{
C. Vaz de Carvalho \\ GILT-ISEP, Rua Dr. António Bernardino de Almeida, 431, P-4200-072 Porto, Portugal
}

\section{Abstract}

The first number of the second volume of the EAI Transactions on Serious Games focuses on the results presented on the European Conference on Game-Based Learning. This event, already on the $8^{\text {th }}$ edition, has set standards in terms of presentation of research and practice and in the pointing out of new and future trends in the development of Game-Based Learning. As such, we are quite thrilled to be able to report them here.

Keywords: Serious Games conferences, Serious Games events.

Received on 01 May 2014, published on 22 May 2014

Copyright (C) 2014 C. Vaz de Carvalho licensed to ICST. This is an open access article distributed under the terms of the Creative Commons Attribution licence (http://creativecommons.org/licenses/by/3.0/), which permits unlimited use, distribution and reproduction in any medium so long as the original work is properly cited.

doi: $10.4108 /$ sg.1.2.e1

The European Conference on Game-Based Learning ECGBL, has been a reference forum to exchange ideas and best practice among researchers and practitioners. The 2013 edition took place in Porto, last October, and was another opportunity for the presentation of research, theory, application, practice and validation in this field. The conference partnered with SEGAN - Serious Games Network, a European community of practice that gathers more than 500 individuals interested in this area, providing a valuable synergy.

The contribution of the keynote speakers, Prof. Baltasar Fernández-Manjón, from the Complutense University of Madrid and Dr Paulo J. Gomes, from Bigmoon Studios, were paramount by addressing research and practical aspects of GBL. But above all, it was the very high number of contributions ranging from theoretical and empirical studies, work-in-progress, $\mathrm{PhD}$ research, to product demonstrations and a game competition that created the conditions for the success of the conference. From that large number of contributions, we selected five that demonstrate the quality of the overall conference and the large scope of targets, topics, technologies and methodologies that GBL already addresses. These contributions address different perspectives of the use of Serious Games for educational purposes.

Mounier et al address the issue of multi-player learning games and the problems related to their development. They propose a framework that enables a formal modelling and an associated automatic verification of learning game's scenario at the design stage of development process, therefore anticipating potential problems in the development stage.

Oostendorp et al focus on the dynamic adaptation of a game as a direct result of the proficiency demonstrated by the player. They found that an adaptable game is significantly more efficient and results in higher learning gains per instructional case, but does not lead to a difference in engagement. Therefore they view games adaptability as a tool to produce more efficient learning tools.

Ali Oulhaci et al present and assess an example of the use of Serious Games for crisis management, based on a multi-agent system architecture supporting behavioural simulation as well as monitoring and assessment of human players.

Another example of the application of Serious Games is brought by Wilson and McDonagh. They focus on young people living with long term health conditions and on the application of gamification (using game mechanics in non-game contexts) to improve the engagement of young people in managing their self-care and provide a mechanism for doctors to quantifying the acquisition of these skills.

Finally, Bratitsis and Kandroudi raise a discussion regarding motion sensor technologies and its exploitation within the educational context. They present an overview of the existing literature and categorize the educational approaches that involve motion sensor technologies namely for people with special needs and for the general public. 